\title{
Classificação de Ambientes com LiDAR-2D para Navegação em Robótica Móvel
}

\author{
Quenaz B. Soares, Waldemiro L. Junior, \\ Alexandre S. Brandão \\ Núcleo de Especialização em Robótica, Departamento de Engenharia \\ Elétrica \\ Universidade Federal de Viçosa, Viçosa - MG \\ (e-mail: quenaz.soares@ufv.br,waldemiro.junior@ufv.br, \\ alexandre.brandao@ufv.br).
}

\begin{abstract}
In mobile robotics, techniques for identification and classification of environments aim to assist in route planning. In this context, this work addresses the classification of environments through neural networks applied to readings of a $2 \mathrm{D}$ LiDAR on board a mobile robot. The proposed strategy presented a hit rate of more than $90 \%$ for the differentiation among room, open area, narrow corridor and wide corridor. The proposed system presents low computational cost, being a powerful and promising tool to assist in the local planning of navigation strategies.

Resumo: Em robótica móvel, técnicas de identificação e classificação de ambientes visam auxiliar no planejamento de rotas. Neste contexto, este trabalho aborda a classificação de ambientes por meio redes neurais aplicadas a leituras de um LiDAR 2D a bordo de um robô. A estratégia proposta apresentou uma taxa de acerto superior a $90 \%$ para a diferenciação entre sala, área aberta, corredor estreito e corredor largo. O sistema proposto apresenta baixo custo computacional, sendo uma ferramenta poderosa e promissora para auxiliar no planejamento local de estratégias de navegação.
\end{abstract}

Keywords: mobile robotic; LiDAR; environment classification; neural network; local planning. Palavras-chaves: Robótica móvel, LiDAR, classificação de ambiente, rede neural, planejamento local.

\section{INTRODUÇÃO}

O aumento das pesquisas em robótica vem influenciando cada vez mais a vida humana. Dentre seus vários segmentos, encontra-se a robótica móvel, a qual tem o papel de desenvolver agentes inteligentes capazes de se locomover em ambientes dinâmicos, estáticos, conhecidos ou não, de forma autônoma e eficiente (Siegwart et al., 2011).

Para atender a essa demanda, há um interesse em se obter uma rota ótima e livre de colisão durante a navegação. Dentre inúmeras formas analíticas, empíricas ou probabilísticas de solucionar este problema, a Inteligência Artificial (IA) atende esse propósito e é um dos métodos mais utilizados nesse ramo, através da aplicação de técnicas como lógica fuzzy, redes neurais artificiais (RNAs), algoritmos genéticos, ou até mesmo uma combinação dessas estratégias (Martinez-Soto et al., 2012; Juang and Chang, 2011).

No tocante a navegação de robôs, diversos autores têm utilizado técnicas para produzir caminhos eficientes em ambientes estáticos e dinâmicos. Na maior parte da literatura, essas técnicas aplicam planejamento global, cujas rotas ótimas são obtidas a um alto custo computacional ou planejamento local, onde rotas subótimas são determi-

\footnotetext{
* Suporte financeiro: FAPEMIG e CNPq.
}

nadas a um custo computacional mais baixo (Altaharwa et al., 2008; Shamsinejad et al., 2010; Yun et al., 2011; Shi and Cui, 2010; Tuncer and Yildirim, 2012).

Por outra perspectiva, existem estudos voltados para o mapeamento do ambiente e detecção de obstáculos, durante a navegação do robô. Para isso, é possível utilizar sensores de percepção como câmeras e LiDAR. Estes são usados para criar um modelo interno do ambiente permitindo a localização e o planejamento de rotas navegáveis do terreno, definidas ou rotuladas por algum classificador (S.Thrun et al., 2005).

É importante ressaltar que, para a navegação, o robô deve planejar uma rota evitando obstáculos e regiões que não são apropriadas. Daí a necessidade de mapeamento do ambiente. Na literatura, para representação em 3D, os métodos mais utilizados são a nuvem de pontos, malhas triangulares e estruturas planas, os quais requerem mais espaço de armazenamento quanto maior o ambiente, tornando-se, assim, uma desvantagem desse método. Em contrapartida, os mapas em 2D, por serem representados por grades de células, utilizam menos recursos computacionais e são eficientes para representar caminhos percorríveis (S.Thrun et al., 2005).

A.Hata et al. (2009) apresenta um trabalho de classificação de ambiente baseado em redes neurais artificiais multi- 
layer perceptron, cujos rótulos de cada célula de navegação são ambiente navegável, parcialmente navegável e não navegável. Ainda no tema de representação, a técnica de Ye and Borenstein (2003) gera mapas precisos do terreno utilizando um LiDAR 2D e realiza a filtragem para remoção de pixels corrompidos e de dados perdidos, a fim de atenuar ruídos nesses mapas. Também utilizando um LiDAR, Bok et al. (2007) cria mapas 3D de estruturas urbanas associando imagens capturadas por uma câmera com os dados fornecidos pelo sensor. Por sua vez, Thrun et al. (2000) utiliza um sensor a laser para possibilitar, em tempo real, a construção de mapas tridimensionais de ambientes cíclicos.

$\mathrm{Na}$ navegação exterior, seja em ambientes semi ou não estruturados, é importante determinar formas seguras de percorrer uma rota. Para tal, existem outras obras dedicadas à identificação de ambientes navegáveis como, por exemplo, a proposta de Wolf et al. (2005), onde um mapeamento do ambiente é feito combinando dados de um odômetro e uma unidade de medição inercial (IMU, do inglês Inertial Measurement Unit). A classificação é realizada da seguinte forma: terrenos planos são classificados como navegável e terrenos irregulares são classificados como não navegáveis baseada no modelo oculto de Markov.

No contexto apresentado, este presente trabalho propõe uma forma simples e eficiente de identificação de ambientes, que possa ser implementada em um sistema de planejamento local de rotas de navegação. A entrada para o sistema de classificação são leituras 2D de distâncias fornecidas por um sensor LiDAR, e as classes de saída são: área aberta, sala, corredor estreito e corredor largo. Vale ressaltar que, apesar de estar fora do escopo deste trabalho, a definição da classe será útil para a decisão da estratégia de navegação a ser adotada.

O trabalho está organizada da seguinte forma: A Seção 2 descreve o processo de aquisição, lista os rótulos estabelecidos para os ambientes e descreve o processo de classificação e filtragem dos resultados. A Seção 3 apresenta os resultados da classificação direta e aponta uma caminho para melhorar a resposta do classificador através de uma avaliação temporal. Por fim, a Seção 4 apresenta as considerações finais do trabalho ressaltando a viabilidade da proposta para utilização embarcada em robôs com baixa capacidade de processamento.

\section{MATERIAIS E MÉTODOS}

\subsection{Aquisição de dados}

O sensor utilizado neste trabalho foi o LiDAR modelo LHS111 da marca Sick, com um ângulo de abertura horizontal de $270^{\circ}$, resolução angular de $0,5^{\circ}$, frequência de varredura de $50 \mathrm{~Hz}$ e uma faixa de trabalho de $0.5 \mathrm{~m}$ a $20 \mathrm{~m}$. A frequência de amostragem foi ajustada para $5 \mathrm{~Hz}$, de modo a não gerar diversas leituras muito similares em sequência e não comprometer o processo de classificação. Além disso, a baixa frequência de amostragem também auxilia na redução do custo computacional do sistema.

O robô utilizado foi o modelo Pioneer P3 DX, um dos mais populares do mundo para estudos científicos e trabalhos acadêmicos nas universidades. Adequado para ambientes de laboratórios, o P3 DX é um robô móvel de tração diferencial, que possui uma velocidade máxima de $1.6 \mathrm{~m} / \mathrm{s}$ em pisos planos e, em velocidades lentas, pode carregar em sua base até $23 \mathrm{~kg}$ de peso adicional. O Robô e o LiDAR que foi acoplado são exibidos na Figura 1.

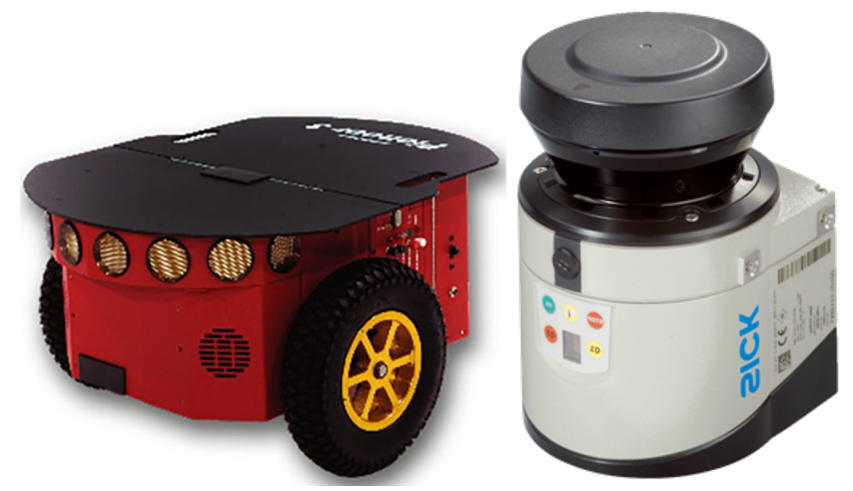

Figura 1. Pioneer 3DX e LiDAR 2D utilizados (sem escala).

\subsection{Ambientes avaliados}

Os ambientes a serem classificados neste trabalho foram divididos em quatro classes, definidas de acordo com os diferentes tipos de rotas e estratégias que, comumente, podem ser adotadas durante a navegação em um ambiente semiestruturado. Tais classes são definidas na Tabela 1 , juntamente com um tipo de estratégia de navegação que se adaptaria a cada ambiente.

Foram coletados dados de alguns ambientes de prédios da Universidade Federal de Viçosa, conforme a Tabela 2. A utilização de prédios diferentes auxilia na generalização dos resultados obtidos, visto que cada prédio possui características distintas para os mesmos tipos de ambientes. Também foram utilizados dados simulados para incluir situações de diferenças tênues entre corredores, sendo simulações de corredor estreito e largo, com 1,4 e 1,6 metros de largura, respectivamente. Dados simulados também foram utilizados para representar salas, neste caso foram utilizadas salas com três dimensões distintas $(2,7 \mathrm{~m} \mathrm{x} 4$ $\mathrm{m} ; 3,8 \mathrm{~m} \times 4 \mathrm{~m}$ com portas e $3,7 \mathrm{~m} \mathrm{x} 4 \mathrm{~m}$ sem portas),

Tabela 1. Descrição das classes de ambientes.

\begin{tabular}{|c|c|c|}
\hline Classe & Características & Estratégia de Navegação \\
\hline Sala & $\begin{array}{l}\text { Ambiente com todas dimensões inferiores a } 5 \text { metros e comprimento } \\
\text { inferior a } 1.5 \mathrm{x} \text { largura }\end{array}$ & Próximo ao centro \\
\hline Área Aberta & Ambientes com todas dimensões superiores a 5 metros & Livre \\
\hline Corredor Largo & $\begin{array}{l}\text { Ambiente com largura entre } 1,5 \text { e } 3 \text { metros e comprimento superior a } 4,5 \\
\text { metros }\end{array}$ & Próximo a uma das laterais \\
\hline
\end{tabular}


Tabela 2. Quantidades de amostras por classe e prédio.

\begin{tabular}{l|llll}
\hline \multirow{2}{*}{ Prédio } & \multirow{4}{4}{ Classes } \\
& Área & $\begin{array}{l}\text { Corredor } \\
\text { Estreito }\end{array}$ & $\begin{array}{l}\text { Corredor } \\
\text { Largo }\end{array}$ & Sala \\
\hline C. Vivência & 2700 & - & 3600 & - \\
CCE & 1800 & 5100 & 8100 & - \\
CEE & 1800 & - & 5100 & - \\
DEF & 1200 & - & 3600 & - \\
DEQ & 2100 & 4200 & 5400 & - \\
Labenge & 1800 & 10200 & 10500 & 1200 \\
Bernardão & - & 4500 & 12300 & - \\
DED & - & - & 3600 & - \\
Simulação & - & 4900 & 3810 & 10300 \\
\hline
\end{tabular}

vazias ou com obstáculos. A Tabela 2 exibe a quantidade de leituras realizadas e simuladas para cada ambiente e o prédio onde foram realizadas.

É importante salientar que todas as coletas foram realizadas em situações normais de navegação, onde o robô poderia se deparar com obstáculos e trânsito de pedestres e cargas. O trajeto em todos os ambientes contém trechos em zigue-zague e trechos paralelos a alguma parede, a fim de se ter uma caracterização mais fiel do ambiente, independente da orientação do robô.

\subsection{Classificação}

Devido ao grande ângulo de visão do LiDAR, foi escolhido avaliar a capacidade de classificação a partir de leituras instantâneas, ou seja, não foram utilizados dados para montar um mapa e, posteriormente, para classificar o ambiente mapeado. Esta abordagem é útil para diminuir o esforço computacional, visto que não será realizado nenhum tipo de tratamento dos dados e, portanto, os cálculos serão apenas aqueles inerentes da rede neural.

Foram testadas redes com 5, 10, 15 e 20 neurônios na camada oculta. A entrada é constituída das leituras das distâncias, sendo que foram testadas redes que utilizam todas as leituras e também que utilizam as mesmas leituras, porém, subamostradas com espaçamento de 1, 2, 5, 10, 20, 30 e 45 graus. Cada configuração de neurônios e espaçamento foi treinada e avaliada 10 vezes para reduzir a incerteza devido à atribuição de valores aleatórios aos pesos iniciais das redes.

A toolbox Neural Network Pattern Recognition tool do Matlab foi utilizada para realizar a classificação dos dados. A topologia feedforward com quatro saídas de valores entre 0 e 1 foi utilizada, sendo que a saída com maior valor é considerada a classe identificada pela rede neural.

\subsection{Análise temporal}

Como este trabalho limitou-se a classificar os ambientes detalhados na Tabela 2, porém é sabido que outras classes poderiam ser incluídas, uma vez que são recorrentes em edificações, tais como, cruzamentos, intersecções, bifurcações, confluências e entroncamentos.

Durante a navegação em um corredor contendo portas abertas ou cruzamentos, o classificador sempre fornece como saída alguma das quatro classes da Tabela 1, uma vez que classes adicionais não foram especificadas. Nestes instantes, é esperado que ocorra uma variação (ou chaveamento) na classificação final da rede neural, visto que o ambiente não se encaixa em nenhuma das saídas padrões.

Para verificar a validade dessa suposição foi realizada uma análise nos trajetos retilíneos (paralelos a alguma parede) e da saída do classificador. A proposta foi verificar após quantas classificações pode-se assumir a seleção de um dado ambiente. Para ilustração, a Figura 2 mostra a saída do classificador para $n=1$ (instantânea), e $n=$ 3 (resposta após três confirmações consecutivas de uma classe). É notório que o efeito de chaveamento entre classes é minimizados, porém há um atraso de $n-1$ amostra para definição da resposta de classe.

Assume-se que os ambientes reais de navegação não possuem variações de classes, conforme ilustrado na parte superior da Figura 2. Logo, a classificação por análise temporal janelada é uma aproximação factível.
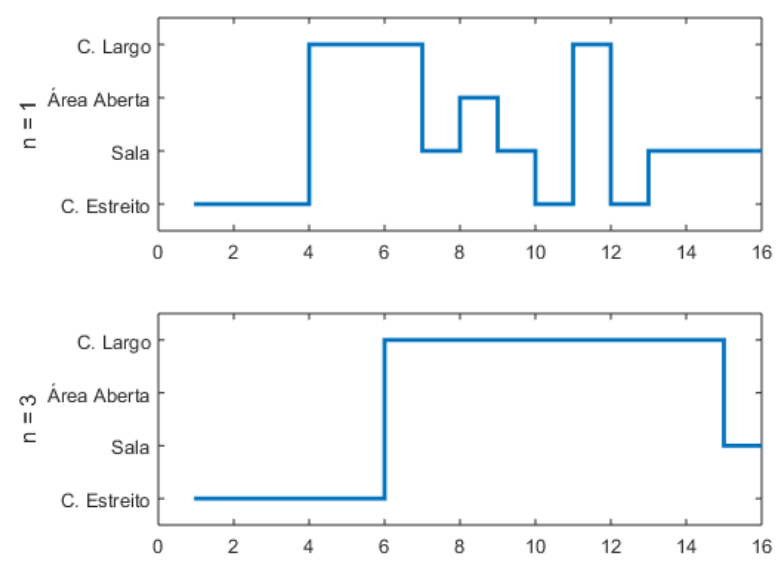

Figura 2. Pós-processamento por análise temporal, em uma janela com 16 amostras.

\section{RESULTADOS E DISCUSSÃO}

\subsection{Desempenho das redes neurais}

Após o treinamento foi avaliado o desempenho das redes utilizando os dados como um todo, ou seja, foram utilizados os dados de treinamento, validação e teste para estimar o desempenho geral da rede.

Os resultados obtidos para cada configuração de número de neurônios na camada oculta e espaçamento angular são apresentados na Tabela 3. Para verificação de diferença estatística entre as configurações, foi utilizado o teste $t$ pareado entre cada par de configuração. Para o número de neurônios escolhidos, todas configurações avaliadas são estatisticamente diferentes para níveis de significância de até $1 \%$, com p-valores de cada comparação apresentados na Tabela 4.

Como as configurações de neurônios são estatisticamente diferentes, pode-se afirmar que utilizar 20 neurônios é melhor, já que é a configuração com maior média de desempenho. A análise do espaçamento foi realizada apenas para a configuração de 20 neurônios na camada oculta. Para comparar estas configurações foi utilizado o teste de Tukey, 


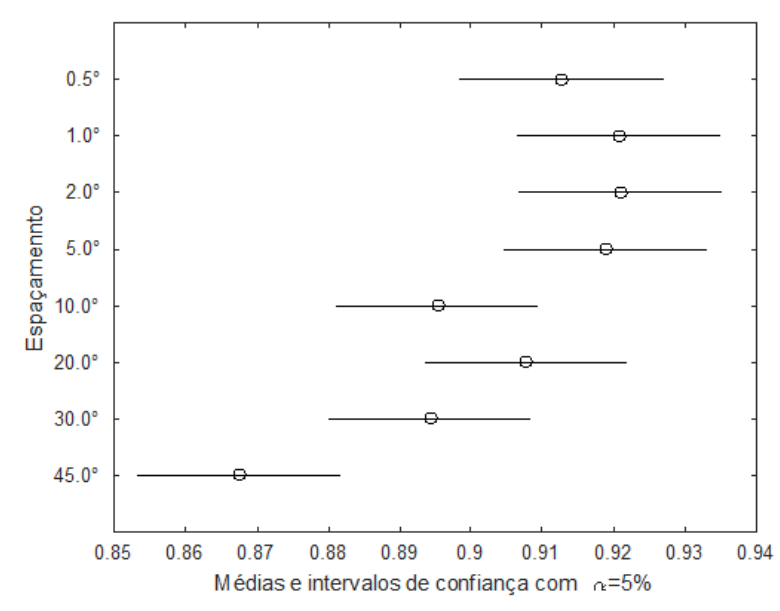

Figura 3. Gráfico do teste de Tukey para comparação dos espaçamentos utilizando a rede neural com 20 neurônios na camada oculta.

que essencialmente se trata de uma representação gráfica de um teste t, o gráfico gerado por esse teste é mostrado na Figura 3. Apesar da significativa taxa de acerto superior dos intervalos de $1^{\circ}, 2^{\circ}$ e $5^{\circ}$, não existe diferença estatística entre os espaçamentos, exceto para $45^{\circ}$. Logo não se pode afirmar que existe algum tipo de ganho em desempenho ao utilizar grande resolução angular nos dados do laser. Portanto, levando em consideração o gasto computacional, optou-se por utilizar um espaçamento de $30^{\circ}$, pois serão apenas 9 entradas para a rede neural.

\subsection{Análise temporal}

Para a realização da análise temporal desconsidera-se as análises estatísticas e utiliza-se a configuração que gerou a rede neural com maior desempenho dentre todas as repetições. Tal configuração consiste de um espaçamento de $5^{\circ}$ e 20 neurônios na camada oculta e a matriz de confusão resultante é mostrada na Figura 4. As classes enumeradas na matriz são: 1 - corredor estreito, 2 - sala, 3 - área aberta e 4 - corredor largo. Nesta análise foram utilizados apenas os dados em que o robô descreveu um percurso retilíneo, pois essa é uma situação mais próxima das que ocorrem durante uma aplicação real.

A partir desta análise foi possível identificar que mesmo durante a execução de uma rota retilínea ao longo do meio de um corredor há uma alteração da classe identificada, isso se deve à existência de obstáculos, principalmente armários, portas abertas e interseções de corredores, os quais alteram as características do ambiente analisado. A Figura 5 mostra uma análise temporal do ambiente de corredor estreito exibido na Figura 6, aplicando o algoritmo de tratamento proposto. Quando $n=1$ temse a saída não tratada da rede neural. Note que ocorrem diversas classificações individuais como corredor largo, afinal esse ambiente tem algumas portas abertas espaçadas entre si e uma região na qual ocorrem três portas em sequência.

A Figura 7 mostra uma leitura dessa região que foi classificada como corredor largo. O algoritmo proposto foi capaz de lidar com as oscilações decorrentes das passagens diante da maioria das portas utilizando uma retenção

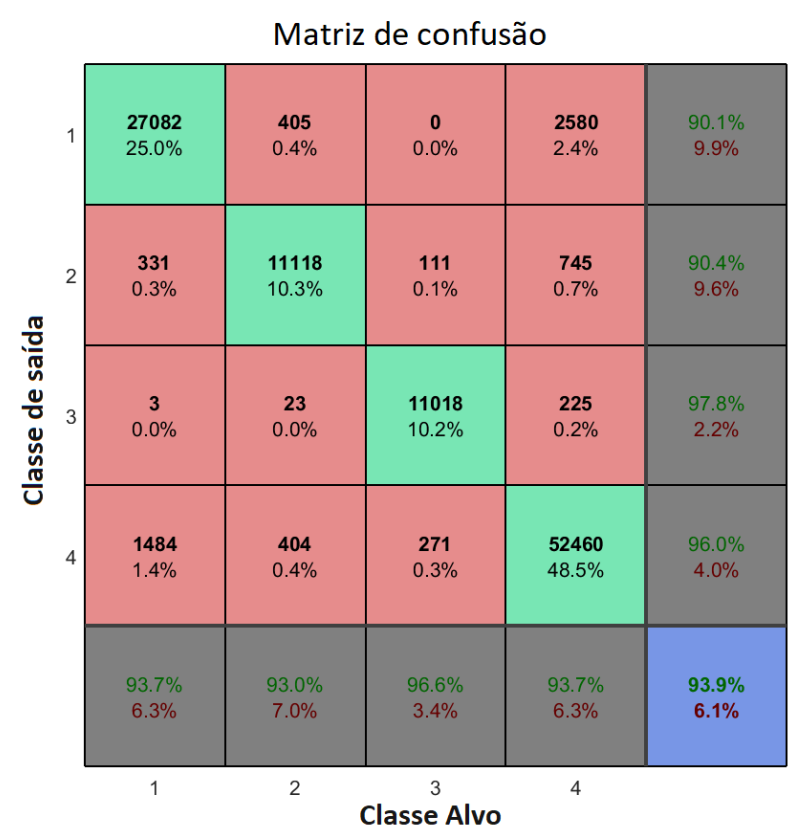

Figura 4. Matriz de confusão da melhor rede obtida, para todos os dados.
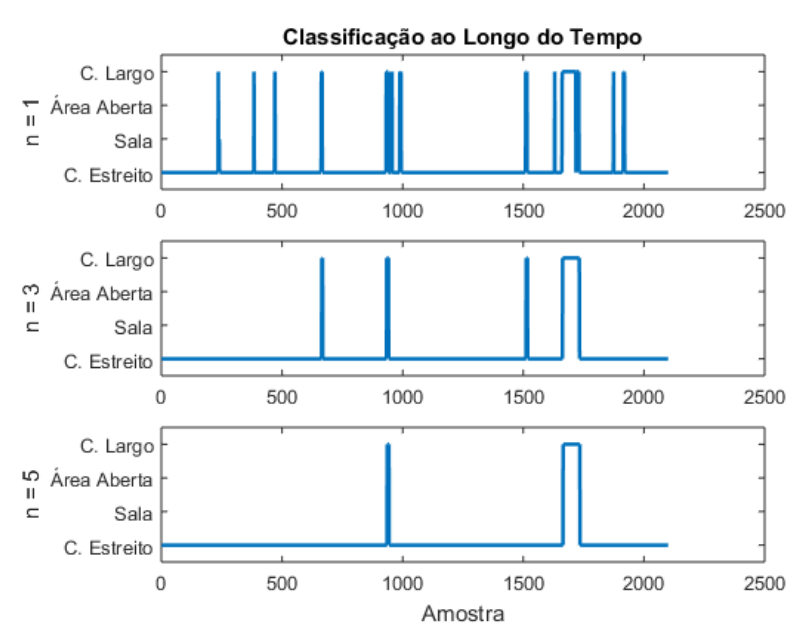

Figura 5. Análise temporal de um corredor estreito contendo obstáculos, portas abertas cruzamentos.

de apenas 5 amostras para classificação definitiva, com exceção da região com as três portas em sequência.

A Figura 8 mostra a análise temporal de um corredor largo com diversos obstáculos, incluindo armários, equipamentos de laboratório e um grupo de pessoas. O algoritmo proposto foi capaz de eliminar a grande variação na saída do classificador, os erros neste ambiente são decorrentes de erro na rotulagem proposta, pois, em diversos pontos do corredor, a largura é reduzida de modo que poderia ser classificado como corredor estreito, como é possível notar na Figura 9. Logo, neste ambiente, o maior ganho do algoritmo proposto é a redução na variação da saída, mantendo uma classificação próxima do ideal, inclusive em regiões de transição temporária de classes de ambientes.

Ainda da análise temporal foi possível observar que ao chegar ao final de corredores estreitos o classificador tende 
Tabela 3. Desempenho das redes neurais aplicadas ao banco de dados. Representados na forma: média (desvio padrão).

\begin{tabular}{|c|c|c|c|c|c|c|}
\hline \multicolumn{7}{|c|}{ Número de neurônios na camada oculta } \\
\hline \multirow{10}{*}{ 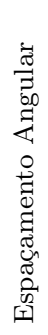 } & & 5 & 10 & 15 & 20 & Média \\
\hline & $0,5^{\circ}$ & $82.9(3.8)$ & $87.1(2.5)$ & $89.9(1.8)$ & $91.3(1.3)$ & $87.8(4.1)$ \\
\hline & $1,0^{\circ}$ & $82.4(2.5)$ & $86.7(3.2)$ & $89.8(2.9)$ & $92.1(1.0)$ & $87.8(4.4)$ \\
\hline & $2,0^{\circ}$ & $81.9(3.0)$ & $89.0(2.9)$ & $90.7(0.9)$ & $92.1(1.3)$ & $88.4(4.5)$ \\
\hline & $5,0^{\circ}$ & $82.0(3.8)$ & $88.8(3.6)$ & $89.9(4.0)$ & $91.9(1.4)$ & $88.2(5.0)$ \\
\hline & $10,0^{\circ}$ & $79.2(3.4)$ & $88.7(2.6)$ & $91.4(0.8)$ & $89.5(4.9)$ & $87.2(5.7)$ \\
\hline & $20,0^{\circ}$ & $79.3(5.9)$ & $85.8(7.0)$ & $89.7(1.3)$ & 90.8( & $86.4(6.4)$ \\
\hline & $30,0^{\circ}$ & 78.6 & 84.6 & 88.5 & 89.4 & 85.3 \\
\hline & $45,0^{\circ}$ & $76.2(5.6)$ & $84.8(1.6)$ & $86.4(1.0)$ & $86.8(1.0)$ & $83.5(5.2)$ \\
\hline & Média & $80.3(4.5)$ & $86.9(4.0)$ & $89.6(2.4)$ & $90.5(2.6)$ & \\
\hline
\end{tabular}

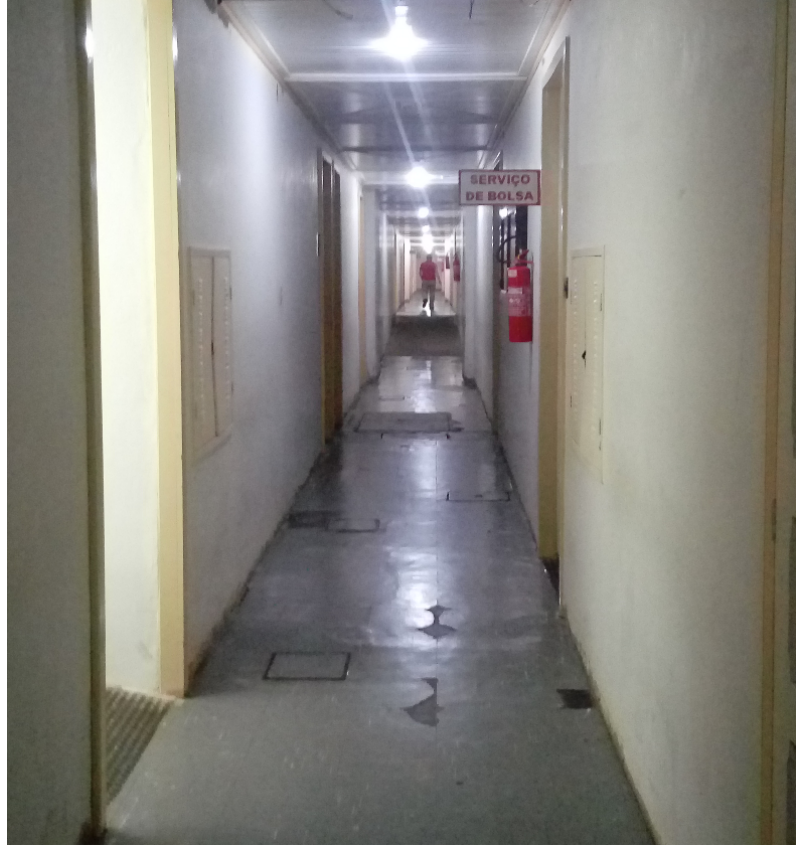

Figura 6. Foto do corredor estreito analisado na Figura 5

a ter como resultado uma sala, visto que o sensor obtém dados de um ambiente fechado, cujas dimensões são aquelas estabelecidas para uma sala, conforme Tabela 1. Além disso, em áreas envidraçadas, os ambientes tendem a ser classificados como áreas abertas, pois o laser retorna uma distância indeterminada nessas situações, cujo valor é idêntico ao retornado para distâncias superiores ao limite de leitura do mesmo. Logo a leitura de uma sala completamente envidraçada é idêntica à de um campo aberto e, assim, a classificação final deve ser a mesma.

Tabela 4. Valor-p do teste t comparando as médias de cada par de configuração de número de neurônios na camada oculta. Pares denotados por $(a, b)$.

\begin{tabular}{cc}
\hline Pares & P-valor \\
\hline$(5,10)$ & $1.5541 e-16$ \\
$(5,15)$ & $1.4387 e-2$ \\
$(5,20)$ & $6.5723 e-33$ \\
$(10,15)$ & $1.0965 e-06$ \\
$(10,20)$ & $2.8005 e-10$ \\
$(15,20)$ & 0.0051 \\
\hline
\end{tabular}

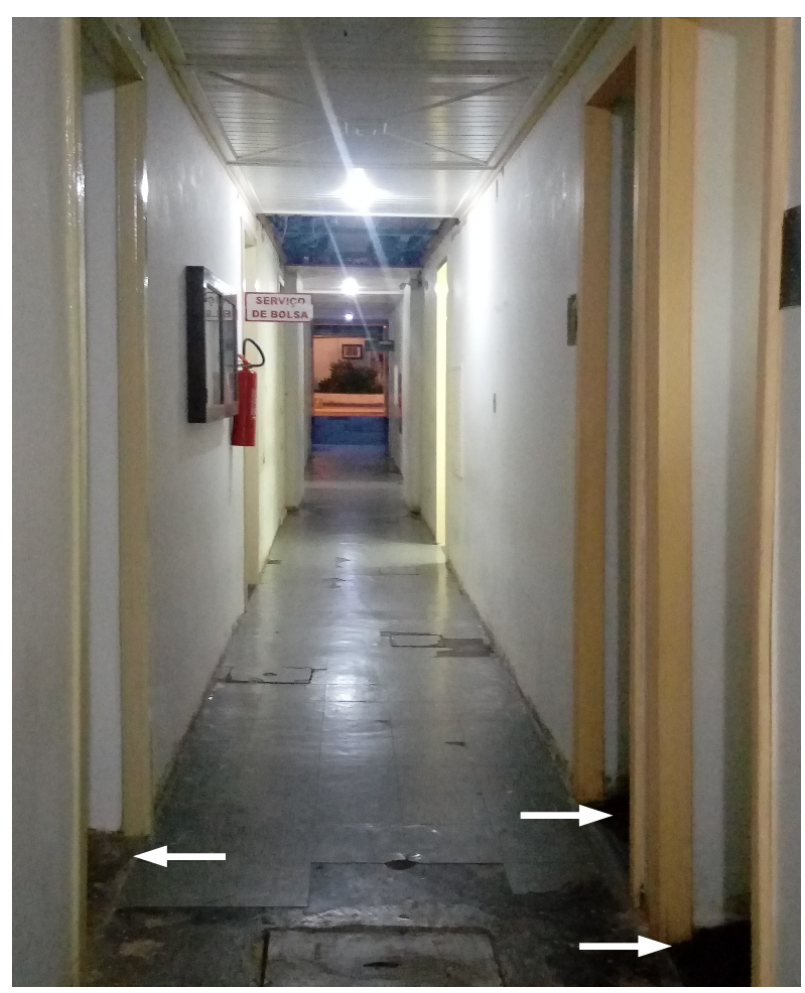

Figura 7. Foto da região do corredor estreito analisado na Figura 5 que apresenta três portas em sequência (evidenciadas pelas setas) e classificação de corredor largo.

\section{CONSIDERAÇÕES FINAIS}

O sistema aqui proposto tem como principal aplicação auxiliar no planejamento local da estratégia de navegação do robô através da identificação do ambiente de navegação.

Uma classificação com desempenho superior a 90\%, associado com um algoritmo de tratamento de saída, fornece uma ferramenta para escolha da melhor estratégia, com baixo custo computacional. A utilização de dados subamostrados também colabora para a redução deste custo, afinal pode-se utilizar até um espaçamento de $30^{\circ}$ sem perda significativa de desempenho.

O sistema completo de identificação possui um desempenho suficiente para sua aplicação e como seu principal componente de classificação é uma rede neural, tem-se algumas vantagens extras, como a possibilidade de aumentar o treinamento e adaptar a rede a ambientes específicos. 

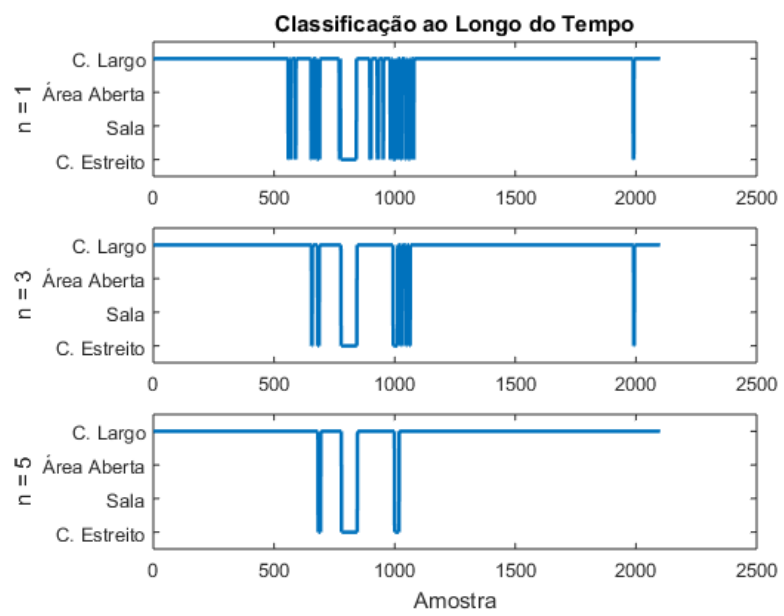

Figura 8. Análise temporal de um corredor largo contendo diversos obstáculos.

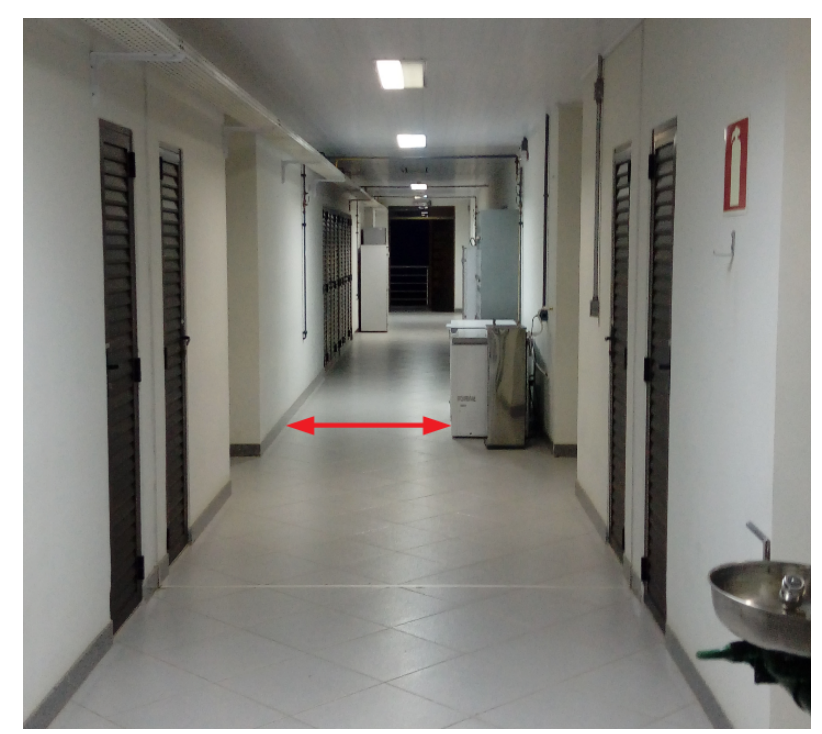

Figura 9. Foto da região do corredor largo analisado na Figura 8, seta indica região com estreitamento.

Ainda existe a possibilidade de realizar um novo treinamento offline com os dados coletados pelo robô durante sua utilização diária, deste modo o treinamento é atualizado com os dados do próprio ambiente de trabalho.

Por fim, o desempenho da rede neural pode variar caso seja necessário identificar outros tipos de ambientes como, por exemplo, cruzamentos, intersecções, bifurcações, confluências e entroncamentos. Logo, uma nova análise de desempenho está em aberto como proposta para trabalhos futuros.

\section{AGRADECIMENTOS}

Os autores agradecem à equipe do Núcleo de Especialização em Robótica da Universidade Federal de Viçosa. Q. B. Soares agradece ao PIBIT/CNPq pela bolsa de iniciação tecnológica. Dr. Brandão agradece ao apoio financeiro da FAPEMIG para a realização deste trabalho.

\section{REFERÊNCIAS}

A.Hata, Wolf, D., Pessin, G., and Osorio, F. (2009). Terrain mapping and classification using neural networks. volume 321, 438-442. doi:10.1145/1644993.1645074.

Altaharwa, I., Sheta, A., and Alweshah, M. (2008). A mobile robot path planning using genetic algorithm in static environment. Journal of Computer Science, 4, 341-344. doi:10.3844/jcssp.2008.341.344.

Bok, Y., Hwang, Y., and Kweon, I.S. (2007). Accurate motion estimation and high-precision $3 \mathrm{~d}$ reconstruction by sensor fusion. In Proceedings 2007 IEEE International Conference on Robotics and Automation, 4721-4726. doi:10.1109/ROBOT.2007.364206.

Juang, C. and Chang, Y. (2011). Evolutionary-groupbased particle-swarm-optimized fuzzy controller with application to mobile-robot navigation in unknown environments. IEEE Transactions on Fuzzy Systems, 19(2), 379-392. doi:10.1109/TFUZZ.2011.2104364.

Martinez-Soto, R., Castillo, O., Aguilar, L.T., and Baruch, I.S. (2012). Bio-inspired optimization of fuzzy logic controllers for autonomous mobile robots. In 2012 Annual Meeting of the North American Fuzzy Information Processing Society (NAFIPS), 1-6. doi:10.1109/NAFIPS. 2012.6291053.

Shamsinejad, P., Saraee, M., and Sheikholeslam, F. (2010). A new path planner for autonomous mobile robots based on genetic algorithm. In 2010 3rd International Conference on Computer Science and Information Technology, volume 8, 115-120. doi:10.1109/ICCSIT.2010. 5563666.

Shi, P. and Cui, Y. (2010). Dynamic path planning for mobile robot based on genetic algorithm in unknown environment. In 2010 Chinese Control and Decision Conference, 4325-4329. doi:10.1109/CCDC.2010.5498349.

Siegwart, R., Nourbakhsh, I., and Scaramuzza, D. (2011). Introduction to autonomous mobile robots. MIT press.

S.Thrun, Burgard, W., and Fox, D. (2005). Probabilistic robotics. MIT press.

Thrun, S., Burgard, W., and Fox, D. (2000). A realtime algorithm for mobile robot mapping with applications to multi-robot and $3 \mathrm{~d}$ mapping. In Proceedings 2000 ICRA. Millennium Conference. IEEE International Conference on Robotics and Automation. Symposia Proceedings (Cat. No.00CH37065), volume 1, 321-328 vol.1. doi:10.1109/ROBOT.2000.844077.

Tuncer, A. and Yildirim, M. (2012). Dynamic path planning of mobile robots with improved genetic algorithm. Computers \& Electrical Engineering, 38(6), 1564 - 1572. doi:https://doi.org/10.1016/j.compeleceng.2012.06.016.

Wolf, D.F., Sukhatme, G.S., Fox, D., and Burgard, W. (2005). Autonomous terrain mapping and classification using hidden markov models. In Proceedings of the 2005 IEEE International Conference on Robotics and Automation, 2026-2031. doi:10.1109/ROBOT.2005.1570411.

Ye, C. and Borenstein, J. (2003). A new terrain mapping method for mobile robots obstacle negotiation. In In Proc. of the UGV Technology Conference at the 2002 SPIE AeroSense Symposium, 21-25.

Yun, S., Parasuraman, S., and Ganapathy, V. (2011). Dynamic path planning algorithm in mobile robot navigation. In 2011 IEEE Symposium on Industrial Electronics and Applications, 364-369. doi:10.1109/ISIEA. 2011.6108732. 